\title{
Maternal risk factors for abnormal placental growth: The national
} collaborative perinatal project Kesha Baptiste-Roberts*1, Carolyn M Salafia2,3, Wanda K Nicholson ${ }^{4,5}$, Anne Duggan ${ }^{6}$, Nae-Yuh Wang7 and Frederick L Brancati ${ }^{7}$

\begin{abstract}
Address: ${ }^{1}$ From the Department of Epidemiology, Johns Hopkins Bloomberg School of Public Health, Baltimore, MD, USA, ${ }^{2}$ From Placental Analytics, LLC, Larchmont, NY, USA, ${ }^{3}$ From Institute for Basic Research, Staten Island, NY, USA, ${ }^{4}$ From the Department of Obstetrics and Gynecology, Johns Hopkins School of Medicine, Baltimore, MD, USA, ${ }^{5}$ From the Department of Population and Family Health Science, Johns Hopkins Bloomberg School of Public Health, Baltimore, MD, USA, ${ }^{\circ}$ From the Department of Pediatrics, Johns Hopkins School of Medicine, Baltimore, USA and ${ }^{7}$ From the Department of Medicine, Johns Hopkins School of Medicine, Baltimore, MD, USA
\end{abstract}

Email: Kesha Baptiste-Roberts* - kbrobert@jhsph.edu; Carolyn M Salafia - carolyn.salafia@gmail.com;

Wanda K Nicholson -wnichol@jhmi.edu; Anne Duggan - aduggan@jhmi.edu; Nae-Yuh Wang - naeyuh@jhmi.edu;

Frederick L Brancati - fbrancat@jhmi.edu

* Corresponding author

Published: 23 September 2008

BMC Pregnancy and Childbirth 2008, 8:44 doi:10.1 186/1471-2393-8-44
Received: 29 May 2008

Accepted: 23 September 2008

This article is available from: http://www.biomedcentral.com/I47I-2393/8/44

(C) 2008 Baptiste-Roberts et al; licensee BioMed Central Ltd.

This is an Open Access article distributed under the terms of the Creative Commons Attribution License (http://creativecommons.org/licenses/by/2.0), which permits unrestricted use, distribution, and reproduction in any medium, provided the original work is properly cited.

\begin{abstract}
Background: Previous studies of maternal risk factors for abnormal placental growth have focused on placental weight and placental ratio as measures of placental growth. We sought to identify maternal risk factors for placental weight and two neglected dimensions of placental growth: placental thickness and chorionic plate area.

Methods: We conducted an analysis of 24,135 mother-placenta pairs enrolled in the National Collaborative Perinatal Project, a prospective cohort study of pregnancy and child health. We defined growth restriction as $<10^{\text {th }}$ percentile and hypertrophy as $>90^{\text {th }}$ percentile for three placental growth dimensions: placental weight, placental thickness and chorionic plate area. We constructed parallel multinomial logistic regression analyses to identify (a) predictors of restricted growth (vs. normal) and (b) predictors of hypertrophic growth (vs. normal).

Results: Black race was associated with an increased likelihood of growth restriction for placental weight, thickness and chorionic plate area, but was associated with a reduced likelihood of hypertrophy for these three placental growth dimensions. We observed an increased likelihood of growth restriction for placental weight and chorionic plate area among mothers with hypertensive disease at 24 weeks or beyond. Anemia was associated with a reduced likelihood of growth restriction for placental weight and chorionic plate area. Pre-pregnancy BMI and pregnancy weight gain were associated with a reduced likelihood of growth restriction and an increased likelihood of hypertrophy for all three dimensions of placental growth.

Conclusion: Maternal risk factors are either associated with placental growth restriction or placental hypertrophy not both. Our findings suggest that the placenta may have compensatory responses to certain maternal risk factors suggesting different underlying biological mechanisms.
\end{abstract}




\section{Background}

Placental structure and function determine the growth trajectory of the fetus. Several studies show that abnormal placental growth is associated with adverse pregnancy outcomes [1-3]. A disproportionately heavy placenta, suggestive of placental hypertrophy, may indicate an adaptive response to an adverse intrauterine environment. Placental hypertrophy may occur in the presence of conditions such as maternal anemia [4], cigarette smoking [5] and lower socio-economic status [6]. Conversely, a disproportionately small placenta may indicate poor nutrient supply to the placenta, or hypoxia resulting in placental growth restriction and subsequently fetal growth restriction [3].

Effects of individual maternal risk factors on placental weight and its ratio to birth weight have been previously studied [5-12]. Placental weight, however, is a gross summary measure, devoid of biologic and mechanistic detail. Multi-dimensional measures of placental growth may provide further insight into the understanding of underlying mechanisms of fetal adaptation and the gestational intervals in which these processes occur. For example, lateral growth of the chorionic plate plateaus in the middle of the third trimester, while thickness of the placenta increases primarily between 30 and 32 weeks gestation [9].

We are not aware of prior studies identifying maternal risk factors for abnormal placental growth where placental growth is assessed using multidimensional measures of growth. We examined three dimensions of placental growth: 1) placental weight; 2) placental thickness; and 3) chorionic plate area, using data from the National Collaborative Perinatal Project (NCPP). The NCPP is a unique US population-based birth cohort that includes maternal sociodemographic, clinical and obstetric data, gross placental measures and histopathology, infant birth characteristics and childhood growth measures up to age 8 . This data provides an excellent opportunity to estimate the influence of maternal characteristics on three dimensions of placental growth in a large population-based sample.

Therefore, our objectives were to 1) determine whether maternal characteristics were associated with multidimensional measures of placental growth and if so, 2) estimate the magnitude of association of maternal factors with placental growth restriction $\left(<10^{\text {th }}\right.$ percentile in placental weight, thickness or chorionic plate area; and placental hypertrophy $\left(>90^{\text {th }}\right.$ percentile for placental weight, thickness or chorionic plate area. We hypothesized that lower socioeconomic status (e.g. annual household income, education) would be associated with increased likelihood of placental growth restriction. We also hypothesized that hypertensive disease would be associated with an increased likelihood of placental growth restriction and anemia and gestational diabetes would be associated with increased likelihood of placental hypertrophy.

\section{Methods \\ Study Population}

The Collaborative Perinatal Project was a prospective cohort study of pregnancy and child health enrolling participants between 1959-1966. It was specifically designed to identify determinants of cerebral palsy and allied neurological defects [13]. A detailed description of the methods is published elsewhere [13]. Briefly, approximately 42,000 pregnant women were enrolled at 12 hospitals across the United States (Baltimore MD, Boston MA, Buffalo NY, Memphis TN, Minneapolis MN, New Orleans LA, New York NY [2 hospitals], Philadelphia PA, Portland OR, Providence RI and Richmond VA). Pregnant women were usually enrolled at their first prenatal visit. Participants were deemed ineligible if they were incarcerated, were planning to move from the area, planned to give the child up for adoption or gave birth on the day they were recruited into the study. Records were not kept for women who refused participation at baseline.

\section{Selection Criteria}

For the present analysis, eligible mothers met the following criteria: 1) either Black or Caucasian 2) gave birth to live-born singletons, 3) no congenital anomaly, and 4) gestational age $\geq 36$ and $\leq 42$ weeks.

\section{Data Collection}

Maternal sociodemographic characteristics (age, education, annual household income, smoking status, race) and parity were obtained by self-report during a personal interview. Maternal age was based on age at entry into the study. Education was based on the number of years of education attained and we categorized mothers as having $<12$ years vs. 12 or more years of education. Annual household income was collected using categories of $\$ 1000$ and we dichotomized annual household income using $<\$ 5000$ vs. $\$ 5000$ or more (equivalent to $\$ 30,000$ in 2005). Mothers were classified as non-smokers, light smokers ( $<1$ pack per day) or heavy smokers $(\geq 1$ pack per day) at the time of the interview. Obstetrical factors were obtained from clinical examination and laboratory testing. Anemia was defined as having hemoglobin $<10.0 \mathrm{~g} /$ $\mathrm{dL}$ or hematocrit $[\mathrm{Hct}]<30 \%$ at any time point during the pregnancy. Mothers were classified as having gestational diabetes if they were diagnosed with diabetes, initiated insulin, received an abnormal glucose tolerance test result, or had a blood glucose $\geq 200 \mathrm{mg} / \mathrm{dL}$ during the pregnancy. Hypertensive disease was defined as having a systolic blood pressure $\geq 160 \mathrm{mmHg}$ or a diastolic blood pressure $\geq 90 \mathrm{mmHg}$ and thus includes women with 
chronic hypertension, pregnancy-induced hypertension and preeclampsia.

Maternal weight and height were measured upon enrollment in the study and mother's weight prior to pregnancy was based on self report. Maternal pre-pregnancy bodymass index (BMI) was calculated as pre-pregnancy weight in kilograms divided by measured height in meters squared. At delivery, just prior to giving birth, mother's weight was measured to determine pregnancy weight gain.

\section{Placental Measurements}

Placentas were collected at delivery and examined by trained pathologists according to a standard protocol [14]. Briefly, the length of the umbilical cord was measured and examined for knots and areas of marked edema. The distance from the base of the cord to the closest placental margin (cord insertion distance) was also measured then the cord was severed at the point of insertion. The largest and smallest diameters were recorded in centimeters and the thickness was measured at the center of the placental tissue by piercing it with a sharp rod calibrated in millimeters. The membranes were then trimmed near the margin of insertion. After removal of the membranes, cord, and any blood clots, the placenta was weighed. We estimated chorionic plate area using the formula for the area $[A]$ of an ellipse

$$
A=\frac{\pi \cdot d_{L} \cdot d_{S}}{4}
$$

where $d_{L}$ is the largest diameter and $d_{S}$ is the smallest diameter.

\section{Outcome Measures}

Our primary outcomes are the three dimensions of placental growth: placental weight, thickness and chorionic plate area. For each of the three dimensions of placental growth studied-placental weight, placental thickness, and chorionic plate area-the growth dimension was classified as 'restricted' if it was $<10^{\text {th }}$ percentile and 'hypertrophic' if $>90^{\text {th }}$ percentile. Growth dimensions that fell within the $10^{\text {th }}$ to $90^{\text {th }}$ percentile were considered 'normal'. We used the distribution of the placental measures in the NCPP sample to derive the percentiles used in the classification of restriction and hypertrophy.

\section{Statistical Analysis}

Descriptive statistics, frequencies for categorical variables and means and standard deviations for continuous variables were calculated. For each of the three placental growth parameters (weight, thickness, and area), we constructed parallel multinomial logistic regression analyses to identify (a) predictors of restricted growth (vs. normal) and (b) predictors of hypertrophic growth (vs. normal). In multivariate models, we included maternal age, income, education, race, smoking status, pre-pregnancy BMI, pregnancy weight gain, parity, anemia, gestational diabetes and hypertensive disease as covariates. Results were robust before and after adjustment, therefore we present only adjusted odds ratios in the tables. Analyses were conducted using STATA statistical software (version 9.0; Stata Corporation, College Station, Texas). We provided $95 \%$ confidence intervals for all estimates. We used a 2-sided Bonferroni-adjusted P value of (0.0045) to test for significance in multivariate models because of the possibility that some associations with the 11 risk factors might have arisen due to chance. The study was approved by the Johns Hopkins Medicine Institutional Review Board.

\section{Results}

Four percent of the subjects enrolled were lost to follow up before delivery. Of 34,345 eligible mothers, 10,921 were excluded from analysis because of missing values for variables of interest. An additional 4 were excluded due to implausible values, leaving an analytic sample of 23,420 mothers. Compared to the participants excluded from the analysis, participants in the analytic sample were slightly older, more likely to be White, and more likely to have annual household income greater than $\$ 5000$ (equivalent to $\$ 30,000$ in 2005 ; all $\mathrm{P}<0.05$ ).

Table 1 summarizes characteristics of 23,420 mothers. The mean age of mothers was 24.5 years. Almost half of the mothers were Black and about half did not complete high school. The majority of the mothers had an annual household income less than $\$ 5000$, equivalent to less than $\$ 30,000$ in 2005. Almost half of the women were current smokers. By present standards, the women were fairly lean with a mean pre-pregnancy body mass index [BMI] of $22.8 \mathrm{~kg} / \mathrm{m}^{2}$. The mean weight gain during pregnancy was $9.7 \mathrm{~kg}$, less than the 11-15 kg recommended today for normal weight women. Most of the women $(71.5 \%)$ had a previous live birth. Anemia was common $(21.5 \%)$, but gestational diabetes was not (1.6\%). About $2 \%$ of the women had hypertensive disease.

The results of the multinomial logistic regression analysis for placental growth restriction are shown in Table 2 . Compared to their counterparts, black women were more likely to have evidence of growth restriction for all three placental growth dimensions examined with relative risk ratios [RRR] ranging from 1.36 to 3.22. Compared to their healthier counterparts, mothers with hypertensive disease were almost twice as likely to produce placentas that were growth-restricted for placental weight and chorionic plate area with RRRs of $1.98 ; 95 \%$ Confidence Interval [CI] $(1.54,2.55)$ and $1.86 ; 95 \% \mathrm{CI}(1.46,2.37)$ respectively. In 
Table I: Selected Characteristics of 23,420 Mother Placenta Pairs in the Collaborative Perinatal Project.

\begin{tabular}{|c|c|}
\hline \multicolumn{2}{|c|}{ Maternal Socio-Demographic Characteristics } \\
\hline Age (years) & $24.5 \pm 5.9$ \\
\hline \multicolumn{2}{|l|}{ Education (years) } \\
\hline$\geq 12$ & II,087 (47.4) \\
\hline$<12$ & $12,333(52.7)$ \\
\hline \multicolumn{2}{|l|}{ Race } \\
\hline White & $12,198(52.1)$ \\
\hline Black & II,222 (47.9) \\
\hline \multicolumn{2}{|l|}{ Annual Family Income } \\
\hline$\geq \$ 5,000 \dagger$ & $6,907(29.5)$ \\
\hline$<\$ 5,000 \dagger$ & $16,513(70.5)$ \\
\hline Smoking Intensity & $22.8 \pm 4.3$ \\
\hline Non-Smoker & $12,350(52.7)$ \\
\hline Light Smoker (< I pack/day) & $7,084(30.3)$ \\
\hline Heavy Smoker ( $\geq$ I pack/day) & $3,986(17.0)$ \\
\hline Pre-pregnancy BMI (kg/m²) & $22.8 \pm 4.3$ \\
\hline Pregnancy weight gain (kg) & $9.7 \pm 5.0$ \\
\hline \multicolumn{2}{|c|}{ Maternal Obstetric Characteristics } \\
\hline Anemia & $5,024(21.5)$ \\
\hline Gestational Diabetes & $360(1.6)$ \\
\hline Hypertensive disease & $543(2.3)$ \\
\hline \multicolumn{2}{|l|}{ Parity } \\
\hline Nulliparous & $6,687(28.6)$ \\
\hline Parous & $16,733(7 \mid .5)$ \\
\hline \multicolumn{2}{|l|}{ Placental Characteristics } \\
\hline Placental Weight (grams) & $438 \pm 92$ \\
\hline Placental thickness (mm) & $21.9 \pm 4.8$ \\
\hline Chorionic plate area $\left(\mathrm{cm}^{2}\right)$ & $247.5 \pm 51.3$ \\
\hline
\end{tabular}

All results presented as $\mathrm{n}(\%)$ or mean $\pm \mathrm{SD}$

† Equivalent to approximately $\$ 30,000$ in 2005 .

contrast, mothers with less than 12 years of education compared to mothers with at least 12 years of education were approximately $20 \%$ less likely to have evidence of growth restriction for all three dimensions of growth. Similarly, mothers with an annual household income less than $\$ 5000$ compared to those with income greater than $\$ 5000$, were $20 \%$ less likely to have evidence of growth restriction for placental thickness. Pre-pregnancy BMI, and pregnancy weight gain both appeared to be protective, showing inverse associations with growth restriction for all three dimensions of placental growth. In addition, mothers with anemia were $33 \%$ and $20 \%$ less likely to have growth restriction for placental weight and chorionic plate area respectively.

Predictors of hypertrophy for all three placental growth dimensions included pre-pregnancy BMI, and pregnancy weight gain. For every unit increase in pre-pregnancy BMI there was a 4 to $10 \%$ increase in the likelihood of placental hypertrophy. Similarly, for every kg increase in pregnancy weight gain, there was a 3 to $7 \%$ increase in the likelihood of placental hypertrophy. Mothers with an annual household income less than $\$ 5000$ were $19 \%$ and $20 \%$ more likely to have hypertrophy compared to mothers with incomes $\$ 5000$ or more for placental weight and chorionic plate area respectively. Mothers with anemia were $50 \%$ and $45 \%$ more likely to have hypertrophy compared to mothers without anemia for placental weight and chorionic plate area respectively. Compared to nulliparous women, parous women were $25 \%$ and $32 \%$ more likely to have hypertrophic growth for placental weight and thickness respectively. Mothers with gestational diabetes were $75 \%$ and $59 \%$ more likely to have hypertrophic growth for placental weight and chorionic plate area respectively. In contrast, after accounting for other sociodemographic factors, black mothers were $20 \%$ to $43 \%$ less likely than their white counterparts to have hypertrophy of all three dimensions of placental growth.

\section{Discussion}

Most maternal risk factors were associated with either a hypertrophic or restrictive adaptive growth response of the placenta. Black race was associated with an increased likelihood of growth restriction for placental weight, thickness and chorionic plate area, but was associated with a reduced likelihood of hypertrophy for these three placental growth dimensions. We only observed an increased likelihood of growth restriction for weight and chorionic plate area among mothers with hypertensive disease. Conversely, anemia was associated with a reduced likelihood of growth restriction for placental weight and chorionic plate area. Pre-pregnancy BMI and pregnancy weight gain were associated with a reduced likelihood of growth restriction and an increased likelihood of hypertrophy for all three dimensions of placental growth examined.

The relationship between Black race and placental growth is similar to that observed with birth weight. Blacks are more likely to deliver low birth weight infants compared to their White counterparts $[15,16]$ and in our study Blacks have a higher likelihood of restriction for placental weight, thickness and chorionic plate area. Placental growth precedes birth weight, and previous studies show a positive relationship between placental weight and birth weight [17]. Therefore one could propose that the relationship between Black race and birth weight may be mediated by placentation and placental function. Further understanding of the relationship between placental growth and factors influencing placental growth may elucidate understanding of the cause of race disparity with respect to birth weight and other birth outcomes.

In our study, after adjustment for confounding factors, we observed an increased likelihood of placental hypertrophy and a decreased likelihood of placental growth restriction based on placental weight and chorionic plate 
Table 2: Multivariate Regression Coefficients for the Association of Maternal Risk Factors with Placental Weight, Thickness and Chorionic Plate Area by Placental Growth

\begin{tabular}{|c|c|c|c|c|c|c|}
\hline & \multicolumn{6}{|c|}{ Placental Growth } \\
\hline & \multicolumn{3}{|c|}{$\frac{\text { Placental Restriction }}{\left(<10^{\text {th }} \text { percentile }\right)}$} & \multicolumn{3}{|c|}{ 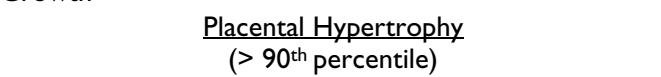 } \\
\hline & $\begin{array}{l}\text { Weight } \\
\text { (g) }\end{array}$ & $\begin{array}{l}\text { Thickness } \\
(\mathrm{mm})\end{array}$ & $\begin{array}{l}\text { Chorionic } \\
\text { Plate Area } \\
\quad\left(\mathrm{cm}^{2}\right)\end{array}$ & $\begin{array}{l}\text { Weight } \\
(\mathrm{g})\end{array}$ & $\begin{array}{l}\text { Thickness } \\
(\mathrm{mm})\end{array}$ & $\begin{array}{c}\text { Chorionic } \\
\text { Plate Area } \\
\left(\mathrm{cm}^{2}\right)\end{array}$ \\
\hline Maternal age (per 5 years) & $1.00(0.99,1.01)$ & $1.02 *(1.01,1.03)$ & $0.99 *(0.98,0.99)$ & $1.01(1.00,1.02)$ & $0.99(0.98,1.00)$ & $1.04 *(1.03,1.05)$ \\
\hline \multicolumn{7}{|l|}{ AnnualFamily Income } \\
\hline$<\$ 5,000$ vs $\geq \$ 5,000$ & $0.88(0.80,0.98)$ & $0.79 *(0.71,0.89)$ & $0.87(0.79,0.96)$ & $1.19 *(1.08,1.32)$ & $0.92(0.83,1.02)$ & $1.21 *(1.07,1.34)$ \\
\hline \multicolumn{7}{|c|}{ 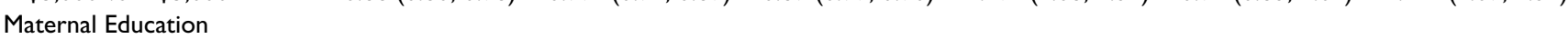 } \\
\hline$<12$ yrs vs $\geq 12$ yrs & $0.83^{*}(0.76,0.91)$ & $0.78^{*}(0.7 \mathrm{I}, 0.86)$ & $0.87 *(0.80,0.95)$ & $1.09(0.99,1.19)$ & $1.15(1.04,1.26)$ & $0.94(0.85,1.04)$ \\
\hline \multicolumn{7}{|l|}{ Maternal Race } \\
\hline Black vs White & $1.66 *(1.51,1.82)$ & $3.22 *(2.90,3.58)$ & $1.36 *(1.24,1.49)$ & $0.57^{*}(0.51,0.63)$ & $0.70 *(0.64,0.77)$ & $0.80 *(0.72,0.89)$ \\
\hline \multicolumn{7}{|l|}{ Smoking Status } \\
\hline Light vs Non-smoker & $0.92(0.84,1.02)$ & $1.03(0.93,1.13)$ & $0.94(0.86,1.03)$ & $0.95(0.85,1.05)$ & $0.89(0.81,0.99)$ & $1.09(0.98,1.21)$ \\
\hline Heavy vs Non-smoker & $1.00(0.89,1.13)$ & I.II $(0.98,1.27)$ & $0.92(0.82,1.03)$ & $0.96(0.85,1.09)$ & $0.85(0.75,0.97)$ & $1.12(0.98,1.27)$ \\
\hline \multicolumn{7}{|l|}{ Parity } \\
\hline Parous vs Nulliparous & $0.89(0.80,0.98)$ & $0.98(0.88,1.10)$ & $1.06(0.96,1.17)$ & $1.25 *(1.11,1.40)$ & $1.32 *(1.18,1.48)$ & $1.09(0.96,1.23)$ \\
\hline \multicolumn{7}{|c|}{ (1... } \\
\hline Yes vs No & $0.67^{*}(0.60,0.74)$ & $1.00(0.91,1.56)$ & $0.80 *(0.72,0.89)$ & $1.50 *(1.34,1.67)$ & $1.18(1.05,1.32)$ & $1.45 *(1.30,1.63)$ \\
\hline \multicolumn{7}{|c|}{ 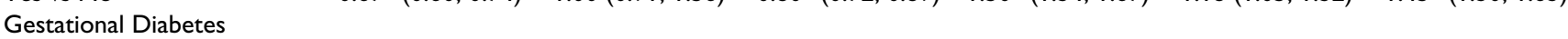 } \\
\hline Yes vs No & $0.77(0.51,1.16)$ & $0.77(0.5 \mathrm{I}, \mathrm{I} .18)$ & $0.68(0.45,1.02)$ & $1.75^{*}(1.33,2.32)$ & $1.29(0.94,1.76)$ & $1.59 *(1.18,2.13)$ \\
\hline \multicolumn{7}{|l|}{ Hypertensive Disease } \\
\hline Yes vs No & I.98* (1.54, 2.55) & $1.19(0.91,1.56)$ & I.86* $(1.46,2.37)$ & $0.62 *(0.46,0.84)$ & $0.69(0.50,0.95)$ & $0.89(0.66,1.19)$ \\
\hline $\begin{array}{l}\text { Pre-pregnancy BMI (per I } \\
\mathrm{kg} / \mathrm{m}^{2} \text { ) }\end{array}$ & $0.91 *(0.90,0.92)$ & $0.98 *(0.97,0.99)$ & $0.93 *(0.92,0.95)$ & $1.10 *(1.06,1.08)$ & $1.05^{*}(1.04,1.06)$ & $1.04 *(1.03,1.05)$ \\
\hline $\begin{array}{l}\text { Pregnancy Weight Gain } \\
\text { (per I kg) }\end{array}$ & $0.93 *(0.92,0.94)$ & $0.99(0.98,1.00)$ & $0.96 *(0.95,0.97)$ & $1.07 *(1.06,1.08)$ & $1.03 *(1.02,1.04)$ & $1.05 *(1.04,1.06)$ \\
\hline
\end{tabular}

$* \mathrm{P}<0.0045$ (Bonferroni-adjusted $\mathrm{p}$-value applied for significance testing)

All results presented as Regression co-efficient (95\% Confidence Interval)

area in the presence of anemia. However, our findings should be interpreted with caution since we were unable to separate anemia resulting from maternal under-nutrition or chronic disease versus pregnancy-related physiologic anemia. The literature on the relationships between maternal anemia and placental weight, and its ratio to birth weight are inconsistent. On the one hand, placental weight and placental ratio appear to increase with severe maternal anemia and this effect has been attributed to maternal under nutrition $[7,12,18-20]$. The thought is that fetal hypoxemia develops consequent to anemia and stimulates growth in order to increase surface area of diffusion exchange and thus offset the impaired oxygen transport [5]. However, there is conflicting evidence showing that placental ratio is lower in the presence of severe iron deficiency anemia [20]. Based on our study results, we are unable to make conclusive statements in support either relationship.

In our study we found that gestational diabetes was associated with an increased risk of placental hypertrophy based on placental weight and chorionic plate area. When we examined the distribution of placental growth meas- ures by gestational diabetes status, we observed an upward shift in the distribution for the subpopulation with gestational diabetes. One explanation for this finding is the increase in the proliferation of peripheral villi and an increase in the length of villi and capillaries observed in the presence of gestational diabetes. This may compensate for the reduced perfusion due the increased vascular resistance [21].

Our study has several strengths. First, to our knowledge, this is the first epidemiologic study to examine the association between maternal risk factors and abnormal placental growth defined using chorionic plate area and placental thickness in addition to placental weight. Second, we were able to assess the relationship between maternal characteristics and placental growth in a population-based sample where the placentas were examined using standardized protocol. Finally, the sample size was large providing great statistical precision.

Several limitations of our study deserve comment. The NCPP is a historical dataset from the 1950's and the management of high-risk obstetrical complications has 
changed substantially over time and cannot be fully adjusted for in multivariate models. However the NCPP represents a large US population-based database that includes maternal variables and detailed placental measurements. Participants excluded from the analysis differed significantly from those included, which introduces some bias. Given the large sample size we suspect this bias is small. Since all women were not routinely screened for GDM during the study time frame, we have likely underestimated the prevalence of GDM in the study sample. We anticipate that an underestimate of GDM prevalence might have limited the power to detect statistically significant associations between GDM and placental growth. Furthermore, the criterion used to define GDM has changed over time which may also have contributed to the low prevalence of GDM observed. Mother's pre-pregnancy weight was obtained by self-report. Although self reported and measured weights tend to be highly correlated [22-25], accuracy may vary significantly according to age and socioeconomic status [23]. In general women tend to underestimate their weight. There was measurement error in the assessment of the placental growth measures. Placental thickness was assessed only at the center of the placenta. There is some variation of placental thickness throughout the placental organ and since thickness represents diffusion conductance, perhaps it would be more representative if thickness was measured at different sites and then a mean thickness calculated. There may be some differential validity in the measurement of largest and smallest diameter with regard to placental shape used in the estimation of chorionic plate area. In addition, our estimate of chorionic plate area assumes that the placenta is shaped as an ellipse; therefore we expect that there may be a slight overestimation.

Finally, we are unable to generalize our results to the general population of today since the sample was enrolled over 40 years ago and was comprised largely of low income African American women. The incidence of maternal risk factors has changed over time. The mothers were leaner and gained less weight during pregnancy than would be expected in a more contemporary cohort.

\section{Conclusion}

Our study results suggest that adverse obstetric conditions are either associated with placental growth restriction or placental hypertrophy, but not both. This implies that the placenta may have specific compensatory responses to adverse maternal obstetric conditions, each with a distinct pathophysiologic mechanism. Further research in a contemporary cohort is warranted to elucidate the biological mechanisms underlying the associations between anemia, gestational diabetes, hypertensive disease, maternal prepregnancy BMI, and pregnancy weight gain with abnormal placental growth and to determine whether abnormal placental growth might mediate effect on birth weight and childhood growth.

\section{Competing interests}

The authors declare that they have no competing interests.

\section{Authors' contributions}

$\mathrm{KB}$ participated in the conception, design and coordination of the study. KB also performed the statistical analysis, interpreted the results and drafted and revised the manuscript. CMS participated in the conception and design of the study and made substantial contribution to the interpretation of study results and critically reviewed the manuscript. WKN provided extensive contribution to the interpretation of data, drafting the manuscript and revising it critically for important intellectual content. AD made substantial contribution with regard to the acquisition of the data and critically reviewed the manuscript. NYW participated in the design and statistical analysis for the data for the study and was involved in drafting the manuscript. FB participated in the conception and design of the study and provided contribution to the interpretation of data, drafting the manuscript and revising it critically for important intellectual content.

\section{Acknowledgements}

The authors are supported by the National Institutes of Health. KBR is supported by the National Heart, Lung and Blood Institute (T32-HL07024).

CMS is supported by K23-MH067857. NW is supported by National Institutes of Health (MOI-RR027 I9) FLB is supported by the National Institute for Diabetes and Digestive and Kidney Diseases (K24-DK62222).

\section{References}

I. Kingdom J, Huppertz B, Seaward G, Kaufmann P: Development of the placental villous tree and its consequences for fetal growth. Eur J Obstet Gynecol Reprod Biol 2000, 92:35-43.

2. Naeye RL: Causes and consequences of placental growth retardation. JAMA 1978, 239:। |45-| |47.

3. Wallace JM, Aitken RP, Milne JS, Hay Jnr WW: Nutritionally-Mediated Placental Growth Restriction in the Growing Adolescent: Consequences for the Fetus. Biol Reprod 2004, 7I(4): I055-1062.

4. Barker DJP, Gluckman PD, Godfrey KM, Harding JE, Owens JA, Beisher NA, Sivasamboo R, Vohra S, Silpisornkosal S, Reid S: Placental hypertrophy in severe pregnancy anemia. J Obstet Gynaecol $\mathrm{Br}$ Commonw 1970, 77:398-409.

5. Wingerd J, Christianson R, Lovitt WV, Schoen EJ: Placental ratio in white and black women: relation to smoking and anemia. Am J Obstet Gynecol 1976, I 24:67I-675.

6. Williams LA, Evans SF, Newnham JP: Prospective cohort study of factors influencing the relative weights of the placenta and the newborn infant. BMJ I997, 3 | 4: I864- I868.

7. Agboola A: Effect of type and duration of anemia on placental weight and villous histology. J Natl Med Assoc 1979, 71:1067-1069.

8. Chakravorty AP: Foetal and placental weight changes in normal pregnancy and pre-eclampsia. J Obstet Gynaecol Br Commonw 1967, 74:247-253.

9. De Souza SW, John RW, Richards B: Studies on the effect of maternal pre-eclamptic toxaemia on placental weight and on head size and birth weight of the newborn. $\mathrm{Br} J$ Obstet Gynaecol 1976, 83:292-298.

10. Hirvonen T, Toivanen P: Parity, Birth and Placental Weight in Normal and Complicated Pregnancy. Acta Obstet Gynecol Scand I97I, 50:67. 
11. Nicholls $V$, Nye EB: The influence of maternal factors on placental weight. NZMed J 1980, 91:426-430.

12. Singla PN, Chand S, Khanna S, Agarwal KN: Effect of maternal anaemia on the placenta and the newborn infant. Acta Paediatr Scand 1978, 67:645-648.

13. Niswander KR, Gordon M: The Women and Their Pregnancies. (NIH). Washington, DC, US Government Printing Office, US Department of Health, Education and Welfare; 1972:73-379.

14. Benirschke K: Examination of the Placenta. Obstet Gynecol I96I, 18:309-333.

15. Alexander GR, Kogan M, Bader D, Carlo W, Allen M, Mor J: US birth weight/gestational age-specific neonatal mortality: 1995-1997 rates for whites, hispanics, and blacks. Pediatrics 2003, III:e6I-e66.

16. Chike-Obi U, David RJ, Coutinho R, Wu SY: Birth weight has increased over a generation. Am J Epidemiol 1996, I44:563-569.

17. Salafia CM, Maas E, Thorp JM, Eucker B, Pezzullo JC, Savitz DA Measures of placental growth in relation to birth weight and gestational age. Am J Epidemiol 2005, 162:99I-998.

18. Beischer NA, Sivasamboo R, Vohra S, Silpisornkosal S, Reid S: Placental hypertrophy in severe pregnancy anaemia. J Obstet Gynaecol Br Commonw 1970, 77:398-409.

19. Hindmarsh PC, Geary MP, Rodeck CH, Jackson MR, Kingdom JC: Effect of early maternal iron stores on placental weight and structure. Lancet 2000, 356:719-723.

20. Rusia U, Bhatia A, Kapoor S, Madan N, Nair V, Sood SK: Placental morphology \& histochemistry in iron deficiency anemia. Indian J Med Res 1988, 87:468-474.

21. Desoye G, Shafrir E: Placental metabolism and its regulation in health and diabetes. Mol Aspects Med 1994, 15:505-682.

22. Bostrom G, Diderichsen F: Socioeconomic differentials in misclassification of height, weight and body mass index based on questionnaire data. Int J Epidemiol 1997, 26:860-866.

23. Kuczmarski MF, Kuczmarski RJ, Najiar M: Effects of age on validity of self-reported height, weight, and body mass index: findings from the Third National Health and Nutrition Examination Survey, 1988-1994. J Am Diet Assoc 200I, I 0 I:28-34.

24. Nieto-Garcia FJ, Bush TL, Keyl PM: Body mass definitions of obesity: sensitivity and specificity using self-reported weight and height. Epidemiology 1990, I:I46-152.

25. Schmidt MI, Duncan BB, Tavares M, Polanczyk CA, Pellanda L, Zimmer PM: Validity of self-reported weight-a study of urban Brazilian adults. Rev Saude Publica 1993, 27:27|-276.

\section{Pre-publication history}

The pre-publication history for this paper can be accessed here:

http://www.biomedcentral.com/1471-2393/8/44/prepub
Publish with Bio Med Central and every scientist can read your work free of charge

"BioMed Central will be the most significant development for disseminating the results of biomedical research in our lifetime. "

Sir Paul Nurse, Cancer Research UK

Your research papers will be:

- available free of charge to the entire biomedical community

- peer reviewed and published immediately upon acceptance

- cited in PubMed and archived on PubMed Central

- yours - you keep the copyright
BioMedcentral 